\title{
«Lire pour résister, lire pour instruire ». La revue de presse de Toussaint Bonvoisin
}

"Read to Resist, Read to Educate." The press Review of Toussaint Bonvoisin

\section{Éric Saunier}

\section{(2) OpenEdition \\ 1 Journals}

Édition électronique

URL : https://journals.openedition.org/ahrf/12871

DOI : $10.4000 /$ ahrf.12871

ISSN : 1952-403X

\section{Éditeur :}

Armand Colin, Société des études robespierristes

\section{Édition imprimée}

Date de publication : 1 septembre 2013

Pagination : 123-144

ISBN : 9782200928261

ISSN : 0003-4436

\section{Référence électronique}

Éric Saunier, « «Lire pour résister, lire pour instruire ». La revue de presse de Toussaint Bonvoisin », Annales historiques de la Révolution française [En ligne], 373 | juillet-septembre 2013, mis en ligne le 01 septembre 2016, consulté le 01 juillet 2021. URL : http://journals.openedition.org/ahrf/12871 ; DOI : https://doi.org/10.4000/ahrf.12871 


\title{
«LIRE POUR RÉSISTER, LIRE POUR INSTRUIRE». LA REVUE DE PRESSE DE TOUSSAINT BONVOISIN.
}

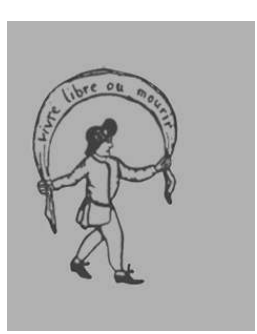

\author{
Éric SAUNIER
}

Octobre 1792 : Toussaint Bonvoisin (1741-1815), un mercier ordinaire retiré dans une maison de la paroisse Notre-Dame (Le Havre), prend la plume. Horrifié par les massacres de Septembre, il commence à écrire un journal, Précis de la Révolution relativement à Louis 16. Respec du aux Rois et aux puissances, dans le but d'instruire ses enfants des méfaits de la subversion spirituelle provoquée par la Révolution. Ce journal long de plus de 4000 pages, qu'il rédige de façon journalière durant un an, entre l'automne 1793 et la fermeture du Club des jacobins en novembre 1794, s'achève au début de 1803. L'étude de cet égo-document, dans lequel sont insérés plus de 200 journaux de la période révolutionnaire, le plus souvent issus de la presse royaliste rouennaise sous le Directoire, permet de voir avec prégnance le rôle que peut jouer la pratique d'une revue de presse dans les écrits des témoins de la crise révolutionnaire. Cette pratique, qui a été jusqu'à ce jour surtout analysée sous l'angle des pratiques collectives, y émerge comme un instrument utilisé pour contourner la difficulté d'écrire née de l'enchaînement de traumatismes entre l'automne 1793 et l'été 1794 et pour améliorer la pédagogie d'un récit dont l'objectif premier est d'ancrer le refus de la Révolution dans la mémoire familiale.

Mots-clefs : Bonvoisin, Directoire, jansénisme, Le Havre, maître de métier, revue de presse

«L'abondance des matieres relativement à la grande conspiration ne nous permettent pas de pouvoir suivre toutes les suittes de cette affaire ;

pour ne rien laisser manquer à l'histoire,

nous nous contentons de joindre à ce précis 
les pièces les plus intéressantes en produisant les journaux tels que tous les rédacteurs rapportent les faits [...]

Le tems nous manquant pour entrer dans le détail de cette affaire, nous donnons ces détails tels qu'ils se trouvent dans les journaux que nous joignons à ce précis »,

\section{Toussaint Bonvoisin,}

21 mai et 12 septembre $1796(\text { XXXIII ; } 16 \text { et } 38)^{1}$

Ces remarques écrites, l'une dix jours seulement après l'arrestation de Babeuf, l'autre au moment de l'échec définitif de la conjuration des Égaux, sont les premières évocations directes que fait Toussaint Bonvoisin de la revue de presse à laquelle il se livre depuis l'automne 1792, soit le moment de démarrage du long journal ${ }^{2}$ qu'il destine à instruire ses enfants des désastres d'une Révolution dont il voulait aussi ancrer le souvenir dans la mémoire familiale ${ }^{3}$. Est-ce la conséquence du sentiment de débordement éprouvé par le mercier havrais face à la difficulté de continuer à relater les troubles intérieurs quand à ce devoir s'ajoute celui de décrire les temps forts de la campagne d'Italie ? Ou est-ce la lassitude qu'il ressent après presque deux années passées à tenter le pari un peu fou d'écrire la Révolution au jour le jour?

Les deux raisons, auxquelles s'ajoute l'effet du soulagement de ce dévot catholique qui avait jugé prudent d'écrire aux Jacobins ${ }^{4}$, en septembre 1793, une lettre pour prévenir d'une possible arrestation, expliquent l'habitude qu'il prend de glisser des pages de journaux dans son propre journal, des articles minutieusement découpés et, de plus en plus au fil

(1) Bibl. Mun. Condorcet (ville de Montivilliers), Ms 71. Suite du Précis de la Révolution française Trente troisieme partie qui comprend depuis le 1 mai 1796 jusqu'au 28 juillet suivant, p. 39 ; Ibid., Trente quatrieme partie... depuis le 1 aout 1796 jusqu' au 28 avril 1797, p. 16.

(2) Cf. Éric SAUNIER, «Un égo-document : le Précis de la Révolution relativement à Louis 16. Respec du aux Rois et aux puissances, $A H R F, \mathrm{n}^{\circ} 365$, juillet-septembre 2011, p. 177-189. Toutes les références indiquées entre parenthèses dans notre texte renvoient aux numéros et aux pages des 42 cahiers du Précis.

(3) Sur ce point, cf. Éric SAUNIER, « Les papiers de la famille Bonvoisin et la mémoire de la Révolution française », Sylvie MOUYSSET (dir.), Entre mémoire et histoire. Écriture ordinaire et émergence de l'individu, Paris, Éditions du CTHS, p. 163-177.

(4) Lettre insérée dans la cinquième partie du Précis, page 21, sous le titre : «Aux Amis de la Constitution et au Comité de salut public, Le 3 de 7bre 1793, L'an $2^{\mathrm{e}}$ de la République françoise une et indivisible ». Signe de la peur de Toussaint Bonvoisin, cette lettre s'achève avec ce post-scriptum : « cette pétition n'a pas été présentée l'arrestation n'ayant eu lieu. Elle avoit été faite toute preste attendant à chaque instant le moment fatal sur les bruits qui s'en débitoient. La Providence par sa sainte Grace nous en a préservées ». 
du temps, des numéros entiers qui sont pour l'essentiel des quotidiens royalistes que publient Magloire Robert ${ }^{5}$ entre le Directoire et le Consulat. Malgré des retours vers l'écriture journalière, comme c'est le cas lors des bombardements anglais du printemps 1798 dont il fut victime ${ }^{6}$, l'insertion des journaux de la Révolution dans le « Précis de la Révolution » devient systématique, au point que le texte est envahi par les numéros de la Chronique de l'Europe après le coup d'état du 18 Brumaire. Lorsque, le 12 janvier 1803, Toussaint Bonvoisin pose la plume, rassuré par la main tendue aux catholiques par Bonaparte et heureux du retour au Havre du prêtre réfractaire Guillaume Anfray ${ }^{7}$ dont le départ fut le véritable déclencheur de l'écriture du Précis, ce sont ainsi plus de 200 journaux - parmi lesquels on voit quelques numéros qui étaient restés méconnus ${ }^{8}$-, une centaine de pages et une cinquantaine d'articles découpés que l'on retrouve, éparpillés, dans les pages de ce témoignage à chaud de la Révolution (Annexe 1).

Cette profusion de journaux, plus encore le fait qu'ils sont l'objet d'annotations, de ratures et découpages qui suggèrent un travail critique, fait de ce texte, à plus forte raison parce qu'il a été écrit sans interruption durant plus de dix ans, un matériau précieux qui permet d'étudier comme on le peut trop rarement les modes d'appropriation de ce nouveau médium. Et l'étude est rendue d'autant plus intéressante que, si l'on en sait un peu de la réappropriation du journal par les élites nobiliaires ${ }^{9}$, celles de ces hommes ordinaires, comme l'est le maître de métier cultivé ${ }^{10}$ Toussaint Bonvoisin, reste encore un territoire en friche.

Comment l'utilisation du journal, à travers la place qu'on lui donne dans le cadre d'un processus d'écriture, peut-elle témoigner de l'impact de la

(5) Cf. Éric WAUTERS, Une presse de province pendant la Révolution française. Journaux et journalistes normands (1785-1800), Paris, CTHS, p. 181-266.

(6) Un témoignage des destructions matérielles subies par la famille Bonvoisin lors du bombardement du Havre le 24 mai 1798 est mentionné dans le Précis : « un boulet a rasé notre maison, a percé la maison de vis à vis et est tombé par l'escalier en bondissant », XXXVIII ; 38.

(7) Le prêtre Guillaume Anfray (1731-1807), figure de proue de la philanthropie et des Lumières au Havre, part à Gosport le 5 septembre 1792. Il engage une correspondance épistolaire régulière avec Bonvoisin dans laquelle le prêtre réfractaire lui demande de réunir la matière nécessaire pour achever un ouvrage commencé avant son départ qu'il va publier sous le titre Les Feuilles civiques. C'est cette fonction de «nègre » qui donna aussi à Toussaint Bonvoisin, parallèlement à l'envie d'instruire ses enfants des méfaits de la Révolution, l'idée d'écrire le Précis.

(8) Le Précis contient quatre numéros de L'Eclipse, alors qu'un seul numéro était connu jusqu'alors

(9) Cf. Marquis de FERRIÈRES, Correspondance inédite (1789, 1790, 1791), éditée par Henri Carré, A. Colin, 1932.

(10) Le constat de la vaste culture de Toussaint Bonvoisin émerge dans sa capacité à se réapproprier les œuvres d'auteurs allant des prédicateurs du XVII ${ }^{\mathrm{e}}$ siècle (Bossuet, Bourdaloue...) aux figures de proue des Lumières catholiques (Caracciolo) en passant par des auteurs étrangers traduits en Français (Edouard Young). 
crise révolutionnaire ? De quelle manière la presse est-elle instrumentalisée par ceux qui, comme ce mercier vivant dans un port colonial ${ }^{11}$, décidèrent de s'en saisir dans l'intimité d'un cabinet d'écriture ? Dans quelle mesure enfin son instrumentalisation dans un contexte de crise politique modifie-t-elle les comportements individuels ? Parce qu'elle apporte quelques réponses à ces questions, l'étude de la revue de presse de Toussaint Bonvoisin et de son utilisation à des fins d'écriture, dont il convient d'interroger le choix, est un fort témoignage de la nécessité d'investir le chantier des égo-documents dans une perspective de renouvellement des études sur la presse révolutionnaire dont Daniel Roche a récemment rappelé qu'elles souffrent encore d'un manque d'autonomie ${ }^{12}$.

Les raisons qui poussent Toussaint Bonvoisin à s'emparer de la lecture des quotidiens pour en faire une pièce de choix dans la rédaction de son journal sont facilitées par le moment et par le lieu de son écriture. En prenant la plume à l'automne 1792, il bénéficie, à la différence de ceux qui avaient décidé de le faire dès l'été $1789^{13}$, des solides positions sociétales acquises par la presse depuis le début de la Révolution. Pour ce bourgeois urbain cultivé, soucieux de trouver « une information complète et fiable » pour instruire ses lecteurs, il est presque naturel de rechercher dans la presse les faits dont il veut dispenser les leçons. La lecture des deux premiers cahiers, dans lesquels il fait la synthèse des événements survenus entre l'ouverture des États généraux et les massacres de Septembre, montre d'ailleurs une propension immédiate à se précipiter pour puiser à la source des meilleurs journaux parisiens les informations nécessaires à la connaissance de la vie parlementaire ${ }^{14}$ naissante. Le Patriote français, malgré l'aversion du mercier envers Brissot ${ }^{15}$, le Défenseur de la Constitution $^{16}$, dont ce catholique, qui défend le principe de la soumission

(11) Pour une présentation de Toussaint Bonvoisin, voir Éric SAUNIER, « Un égo-document... », art. cit. p. 177-189.

(12) Dans Jérémy POPKIN, La presse de la Révolution. Journaux et journalistes (1789-1799), Paris, Odile Jacob, 2011, p. 8.

(13) Cf. Jérémy POPKIN, Ibidem, p. 17-55.

(14) Dès les pages 34/35 de son premier cahier, Toussaint Bonvoisin insère un exemplaire d'une caricature qui est publiée le 2 mai 1790 dans le n²3 des Révolutions de France et de Brabant; «M. le vicomte de Mirabeau voulant escalader la tribune, fait soutenir l'échelle par Mr. l'abbé Maury et Mr. Cazalès mais sa chutte anéantit les aristocrates ».

(15) Aversion due à l'attachement du mercier, qui compte pour ami le porte-parole du lobby négrier Jacques-François Bégouën. Après avoir décrit la violence des journées des 20 juin et 10 août 1792, Bonvoisin écrit : «Brissot dit dans ses $\mathrm{N}^{\circ}$ en parlant de la scène affreuse du 20 juin : ce n'est qu'une petite ondée qui ne fait que refraîchir l'atmosphère politique. Qu'il seroit vil et méprisable celui qui ne se pénétreroit pas de suite de l'horreur de cette phrase assassine » (II ; 3).

(16) On retrouve la copie d'une partie de l'article de Robespierre « Sur le respect dû aux lois et aux autorités », publié dans le $\mathrm{n}^{\circ} 5$ du Défenseur de la Constitution, dans la cinquième partie du Précis, 
au pouvoir établi défendue par Saint-Paul, extrait un article pour justifier en août 1793 la fidélité nécessaire à la Constitution à venir, plus encore les journaux royalistes, La Gazette Universelle et Le Postillon de la Guerre, où ce nostalgique de l'Ancien Régime peut trouver l'abondante matière qui lui est utile pour dénoncer les violences faites au roi lors des journées des 20 juin et 10 août 1792 (I ; 66/99) sont les premiers journaux auxquels il recourt pour entamer un récit qui fait la part belle aux événements parisiens ${ }^{17}$.

L'attitude qui consiste, à l'automne 1792, à s'accaparer la matière profuse des journaux pour instruire de la crise révolutionnaire s'appuie aussi sur l'opportunité offerte par la proximité de Rouen. Vivant au Havre, Toussaint Bonvoisin peut en effet aisément, à côté des journaux parisiens, recourir à l'apport des informations que fournit l'influente presse rouennaise, et particulièrement ses feuilles conservatrices, fort actives depuis l'été $1790^{18}$. De là vient, avec le procès et l'exécution du Roi que Toussaint Bonvoisin présente sur le modèle de la Passion du Christ (III ; $23 / 96 \& 4 ; 1 / 56)^{19}$, son rapide recours au grand journal conservateur rouennais : la Chronique nationale et étrangère. Il présente l'avantage, outre une opposition à la guerre, une volonté sans failles de défendre la royauté ${ }^{20}$ et une sensibilité politique proche de celle de Toussaint Bonvoisin $^{21}$, d'être aussi une feuille attentive à la vie politique parisienne, notamment à l'activité de l'Assemblée constituante et des clubs dont les rédacteurs prennent soin à « rapporter les séances avant les papiers de Paris »

aux pages 83-88. Datée du 12 août, la copie, commentée, porte le titre « Réflexions sur la Constitution républicaine ». La fin du texte de Robespierre est insérée dans le cahier suivant, le 22 septembre, aux pages 48-52. Bonvoisin s'appuie sur ce texte publié dans Le Défenseur de la Constitution, entre le 15 et le 19 juin 1792 pour dénoncer les menaces de La Fayette sur la Constitution de 1791, pour présenter les principes de comportement politique qu'il préconise.

(17) Un regard sur les lieux évoqués dans le Précis montre que les événements parisiens recouvrent presque le même volume $(46 \%)$ que les relations inhérentes aux faits provinciaux, Le Havre inclus.

(18) Cf. Éric WAUTERS, Une presse... op. cit. p. 303.

(19) Exception faite de la longue plaidoirie de Desèze du 26 décembre 1792, qu'il recopie à partir du n ${ }^{\circ} 12$ du Défenseur de la Constitution. (31 décembre 1792).

(20) En témoigne le rôle joué par son rédacteur en chef Leclerc, instigateur le 19 janvier 1793 de la seule manifestation provinciale organisée pour dénoncer la sentence de mort prononcée lors du procès du roi (Éric WAUTERS, Une presse... op. cit.,. p. 128-130).

(21) La sensibilité politique du journal rouennais est proche des feuillants, celle de Bonvoisin des Monarchiens. Celle-ci émerge dans un commentaire de la Fête de la Constitution de 1791 organisée au Havre (manuscrit inséré aux pages 54-55 du premier cahier sous le titre : " Havre. Réjouissance pour l'acceptation de la Constitution ») . 
(II ; 40). Passé le temps des difficultés, relatives, de la période de la Terreur ${ }^{22}$, le dynamisme renouvelé de la presse conservatrice rouennaise durant la période libérale du Directoire - elle bénéfice du repli à Rouen de l'ancien rédacteur en chef du Messager du Soir et de la Gazette Révolutionnaire Magloire Robert-, lui permet de continuer à s'appuyer sans difficulté sur les articles des journaux conservateurs pour stigmatiser la politique gouvernementale et pour rapporter les nouvelles de l'étranger.

Reste que tous les bourgeois havrais cultivés ne furent pas animés de cet intérêt pour la presse, intérêt qui le conduit aussi à s'intéresser au sort des « ouvriers de l'information (J. Popkin) $»^{23}$.

Bien que le temps de l'écriture et la possibilité donnée d'utiliser les richesses d'une presse départementale active soient des facteurs favorables pour utiliser le journal, c'est dans l'appartenance de Toussaint Bonvoisin au milieu janséniste ${ }^{24}$ dont on connaît la capacité à utiliser les Nouvelles ecclésiastiques comme arme de mobilisation militante qu'il faut rechercher les raisons de ce choix immédiat du journal pour alimenter un écrit qui était destiné, comme la feuille janséniste, à exprimer une opposition. Avec la variété de références religieuses caractéristiques de la culture des chrétiens qui puisent leur réflexion aux sources de l'école de spiritualité française, la lecture du texte de Toussaint Bonvoisin révèle, avec l'intérêt qu'il montre pour les écrits de Target - particulièrement pour le texte que l'avocat produisit pour se récuser lors du procès du roi $^{25}{ }^{2}$, avec sa propension à souligner les Mystères de la Religion, avec le respect du prêtre qu'il exprime en brossant les portraits édifiants des réfractaires, avec sa récurrente façon de mobiliser le passé, plus encore avec le choix de copier le Livre XV de l'Histoire ancienne de Rollin ${ }^{26}$ pour écrire, à partir de juin 1793, un second précis destiné à « entrer et faire suite dans l'histoire de la Révolution

(22) La presse de Seine-Inférieure bénéficie sous la Terreur de la fidélité de ce département à la Convention au printemps 1793 et d'une solide réputation de modérantisme (Éric WAUTERS, Une presse... op. cit., p. 154).

(23) Cet intérêt apparaît dans l'attention que porte Toussaint Bonvoisin à l'arrestation des journalistes royalistes Leclerc (à l'hiver 1793), François-Martin Poultier, le rédacteur de L'Ami des Lois et Isidore Langlois, rédacteur du Messager du Soir (sous le Directoire).

(24) Le jansénisme de Bonvoisin a pour origine l'éducation reçue au collège du Havre, établissement fort touché par le projet de refonte exposé dans la Lettre du clergé de France de 1762, et où l'on applique les préceptes du traité des études de Rollin. Voir Albert ANTHIAUME, Le collège du Havre. Contribution à l'histoire de l'enseignement secondaire en France et particulièrement au Havre (1579-1865), Le Havre, 1905, 2 vol., tome 1

(25) Ms $71: \mathrm{I} ; 38 / 45$.

(26) L'influence de L'Epître dédicatoire adressée au duc de Chartres qu'écrit le pédagogue est d'ailleurs, tout autant que les Instructions au Dauphin, que Toussaint Bonvoisin mentionne à deux reprises, celle qui lui donna l'idée d'instruire les enfants à l'aide du Précis. 
française » aux fins de mieux faire comprendre sa lecture providentialiste ${ }^{27}$, une forte sensibilité à l'esprit janséniste. Elle émerge clairement lorsque, le 30 mai 1794, Toussaint Bonvoisin lance une attaque contre la Philosophie, après avoir déjà dénoncé $L$ 'Émile dans un commentaire de l'interdiction du Tombeau des Imposteurs ${ }^{28}$, sous l'intitulé des « Réflexions morales ». Elle désigne la responsabilité du « coriphée des philosophes impies » (III, $31 / 38$ ) et du Vicaire Savoyard ${ }^{29}$ en reprenant point par point l'argumentaire des jansénistes. S'il n'est pas de copie, ni même de référence directe à la presse janséniste dans le journal de Bonvoisin, l'influence de ce courant est donc présente. On le voit aussi dans l'organisation de la vie familiale ${ }^{30}$ et dans ses premiers écrits ${ }^{31}$. C'est de toute évidence lui qui invite Toussaint Bonvoisin à se saisir du journal, après la revue de presse journalière, pour en faire l'outil privilégié de l'écriture de la Révolution.

Le fait n'est pas sans conséquences. Il procure un savoir-faire qui apparaît dans la remarquable capacité d'adaptation de Toussaint Bonvoisin aux conditions nationales de la vie éditoriale (annexe 2). Dès l'automne 1792, on remarque, outre sa capacité à s'appuyer sur l'intense activité des journaux conservateurs rouennais lors du procès du roi, à mettre à profit la complémentarité des journaux. Malgré de fortes affinités avec la presse conservatrice (La Chronique Nationale et Etrangère) où il puise les arguments pour défendre le veto royal, Toussaint Bonvoisin utilise le Journal de Rouen dont il apprécie les éditoriaux et le choix de donner la parole aux lecteurs. Ils sont, selon lui, les meilleurs témoins de la crise ${ }^{32}$. La Terreur survenue, Toussaint sait prolonger cette pratique. En effet, il

(27) . Bibl. Mun. Condorcet : Ms 54, Toussaint Bonvoisin, Histoire d'Alexandre le Grand, fils de Philippe Roy de Macédoine et d'Olympias.

(28) Cf. Suzanne BÉRARD, Le théâtre révolutionnaire de 1789 à 1794. La déchristianisation sur les planches, Paris, PUPW, 2009. Toussaint Bonvoisin considère le contenu du texte de cette pièce comme le résultat de l'apologie de la religion naturelle.

(29) Cf. Monique CotTReT, Jansénismes et Lumières. Pour un autre XVIII e siècle, Paris, Albin Michel, p. 87-111

(30) Né en 1741, Toussaint Bonvoisin voit naître son premier enfant, Louis, premier de 7 enfants nés en l'espace de dix ans, en 1785, alors qu'il est âgé de 44 ans. À bien des égards, la trajectoire familiale de Toussaint Bonvoisin est celle de ces marchands jansénistes restés célibataires pour « servir la cause » avant de fonder une famille (voir Nicolas LYON-CAEN, La boîte à Perrette. Le jansénisme parisien au XVIII e siècle, Paris, Albin Michel, 2011, p. 153-215).

(31) Dans un premier écrit, daté de mai 1791 (Bibl. Mun. Condorcet, Ms 55, Sentiments d'un bon citoyen \& Patriotte -22 mai-4 juin 1791, 54 p-.) où Toussaint laisse éclater sa colère contre le serment ecclésiastique, on remarque «l'omission des jansénistes » parmi les responsables de la Révolution.

(32) Les premiers courriers des correspondants du Journal de Rouen, dont Bonvoisin s'approprie le contenu pour étayer son argumentaire apparaissent, sous la rubrique Réflexions dans le $3^{\mathrm{e}}$ cahier, en novembre 1792. 
sélectionne alors deux feuilles ${ }^{33}$ pour écrire le texte clair et complet qu'il souhaite proposer à ces lecteurs.

Face au Journal de Rouen qu'il juge idéal pour fournir par le biais, d'une part des lettres des communes et de la correspondance des sociétés populaires $^{34}$, d'autre part de la forte présence des nouvelles locales dont les pages sont jugées nécessaires pour montrer les transformations du Havre, les Annales de la Révolution, où les bulletins de la Convention occupent une place de choix, lui permettent d'extraire une matière qui renseigne sur les guerres de Vendée, sur l'insurrection lyonnaise et, la guerre arrivant, sur les grands moments patriotiques que sont la bataille de Fleurus (XVII ; 38/43) et le combat héroïque du Vengeur (XVII ; 58/71) qu'il relate en cédant la parole à Barère. Malgré la rupture que semble provoquer la chute de Robespierre, dans un geste de soulagement, c'est au lendemain de la chute de l'Incorruptible que Toussaint Bonvoisin commence à y insérer les journaux, la capacité à s'adapter au contexte de la vie éditoriale et à exploiter la complémentarité restent de mise jusqu'en 1803. La première se voit dans sa propension à alterner l'utilisation des journaux de Rouen et de Paris dans l'écriture du Précis. Après un bref retour vers les journaux parisiens après le 9 Thermidor, le choix de Robert de faire de Rouen le centre de diffusion pour ses feuilles royalistes ${ }^{35}$ dès le début du Directoire montre la reprise de la stratégie adoptée à l'automne 1792. Puis la crise fructidorienne ${ }^{36}$ le contraint à nouveau à recourir aux journaux parisiens ${ }^{37}$ avant de revenir, à la suite du retour de Robert sur la scène rouennaise après le coup d'état du 18 Brumaire, à la lecture des journaux de Rouen sous le Consulat. Les Affiches de Rouen, La Chronique de l'Europe, titres dont les apports sont complétés par les informations extraites du Courrier de

(33) Quatre feuilles sont alors actives à Rouen, Les Annales de la Révolution, héritier de l'Indicateur du commerce, le Journal de Rouen. Le Flambeau du Républicanisme, journal de sensibilité hébertiste, et Le Postillon du Jour, de sensibilité conservatrice. Les deux derniers sont inutilisés par Toussaint Bonvoisin.

(34) Parmi ces discours des sociétés populaires recopiés, les plus emblématiques de leur utilisation pour dénoncer les transgressions religieuses sont ceux prononcés dans la société populaire de Perpignan, lors des fêtes de la Raison (XIII ; 36/51) et de l'Être Suprême (XVI ; 15/26), que Toussaint Bonvoisin écrit les 30 mars et 30 juin 1794.

(35) Les journaux de Magloire Robert utilisés par Bonvoisin durant cette période sont respectivement : L'Observateur de l'Europe (janvier-octobre 1795), L'Observateur à Rouen (mars-mai 1796), L'Observateur de l'Europe à Rouen (mai 1796-septembre 1797), L'Éclipse (octobre 1796-mars 1797).

(36) Deux des cinq titres provinciaux interdits le 22 Fructidor sont normands : il s'agit du Courrier maritime, journal havrais, et de L'Observateur de l'Europe.

(37) Respectivement L'Ami des Lois, La Gazette Historique (septembre 1797-novembre 1799), Le Bien Informé (septembre 1797-novembre 1799). Sur les journaux sous le Directoire, voir Jerémy POPKIN, « Les journaux républicains (1796-1799) », RHMC, XXXI, janvier-mars 1984, p. 153-167. 
Londres que lui procure l'exilé Anfray ${ }^{38}$, sont en effet les derniers journaux dans lesquels il puise durant cette période pendant laquelle il continue d'apprécier les éditoriaux et les descriptions de la vie politique havraise du Journal de Rouen, le seul titre présent durant les dix années d'écriture du Précis.

Cette maîtrise de la presse laisse penser, à plus forte raison parce que l'auteur du Précis proclame un projet d'instruction dès le début de son journal ${ }^{39}$, que Toussaint Bonvoisin tire profit de sa revue de presse à des fins exclusivement pédagogiques. La perspective pédagogique existe, s'affirme même au fil du temps, mais n'exclut pas un autre usage, plus personnel, lié à une difficulté d'écrire qui peut surprendre chez cet homme animé par une passion de l'écriture qui est telle qu'il décide de prendre la plume quotidiennement après l'exécution de Louis XVI, puis de rédiger des textes volumineux jusqu'à sa mort ${ }^{40}$. Bien que présente dans la vie de Bonvoisin, la passion d'écrire n'exclut pas le « risque du silence » que peut imposer l'impact des événements qui ont suivi le traumatisme, pourtant fondateur de l'écriture, des massacres de Septembre et la dimension contraignante de la tenue d'un journal.

L'effroi ressenti devant le régicide, la peur suscitée par le vote de la Terreur, plus encore le sentiment d'anéantissement éprouvé face à l'onde de déchristianisation, qui nourrit très abondamment ${ }^{41}$ le Précis entre les mois de novembre 1793 et de juin 1794, date à laquelle il relate rien moins que trois Fêtes de l'Être Suprême, à Paris -XVI-30/45-, à Perpignan -XVII ; 15/25- et au Havre -XVII ; 26/34-, sont des raisons solides de lâcher la plume.

La tentation de l'abandon est d'autant plus grande qu'écrire a été pour Toussaint Bonvoisin, du moins pour ses premiers textes écrits en mai

(38) L'envoi du seul « journal français circulant en Angleterre », que dirige l'abbé de Calonne est signalé dans la correspondance que Bonvoisin entretient avec Guillaume Anfray en 1798 Bibl. Mun. (Le Havre). Recueil des Feuilles civiques Ms 524).

(39) Le projet d'instruire ses enfants est évoqué ainsi : « Je reviens à mon but qui n'est que l'analise de nos maux, un précis et non l'histoire entière de nôtre malheureuse révolution. J'écris pour l'instruction de mes enfans, pour l'amour de mon Roi, et pour ma religion que l'on a détruite et chassée ignominieusement. J'écris comme bon catholique, pour faire connoitre le respec et la soumission que l'on doit aux puissances, fussent elles même injustes, comme le dit l'immortel Bossuet » (I ; 24).

(40) Toussaint Bonvoisin semble (probablement) avoir commencé à écrire au printemps 1791, date de rédaction des Sentimens d'un Bon patriote. Il prolonge cette activité bien au-delà du Précis, achevé en 1803. À la veille de sa mort, en 1815, on retrouve dans une « Histoire du Havre » de 300 pages qu'il a débutée juste après le Précis, des notes datées de mars 1814 (Bibl. Mun. Condorcet, Suite de l'histoire du havre de Grace. Ouvrages, agrandissements de la ville. Faits arrivés lors et pendant la Révolution. Troisième partie. Ms 70, p. 54).

(41) Dans les deux cahiers du Précis couvant la période du $1^{\mathrm{er}}$ novembre au 24 décembre, la déchristianisation (lettres d'abdication, descente de cloches..) occupe deux pages sur trois. 
1791 et à partir d'octobre 1792, un acte imposé par les circonstances à un catholique confronté au dilemme que posaient deux devoirs contradictoires. Face à la nécessité de défendre la religion, Toussaint, qui se montre attaché au devoir de soumission du Chrétien aux autorités établies préconisé par les écrits de Saint-Paul ${ }^{42}$ au point de prôner le respect des constitutions révolutionnaires et de désapprouver la Vendée, devait refuser toute forme de rébellion. Répondre à ces deux injonctions laisse peu de choix et, in fine, l'écriture, seule forme de rébellion acceptable, émerge comme une solution pour répondre à ce problème de conscience.

De cet état de fait découlent deux dangers. L'un, lié à l'inévitable désir cathartique, est la déconstruction du projet pédagogique, à laquelle aboutissent, parfois, les formidables explosions de colère lancées contre les figures emblématiques de la déchristianisation ${ }^{43}$ ou la morbidité d'un texte imprégné, à côté des violences religieuses, de la présence du suicide ${ }^{44}$. Le second est ce risque du silence qui, s'il avait triomphé, aurait empêché d'accomplir les devoirs d'instruire et de mémoire auquel Toussaint Bonvoisin avait promis de répondre.

«La plume m'échappe des doigts et se refuse de continuer sur de pareilles barbaries» (II ; 72) «je ne puis me permettre de transcrire tout l'odieux » (VII, 68), « nous n'osons pas donner son discours » (VII ; 72) : les remarques que fait Bonvoisin dès la description des massacres des prisons qui le conduisent déjà à se retirer derrière la copie des pages de Mon agonie de Trente Huit Heures et de l'Almanach des honnêtes gens ${ }^{45}$, puis à

(42) Cette influence sur Bonvoisin émerge dans le choix de copier les versets du chapitre 13 de l'épître de saint Paul aux Romains Obéir aux princes et aux magistrats leur payer ce qui leur est dî,en préambule du Précis (p. 1-14) qu'il conclut ainsi : «Le respec du aux Rois et aux Puissances a été de tout tems un devoir de Religion. Outre que l'apostre St Paul nous la recommande expressément dans son Épistre aux Romains Chap 13-Vet $1^{\text {er }}$ en disant que toute personne soit soumise aux Puissances Supérieures, il n'i a point de Puissance qui ne provienne de Dieu \& et nous en voions des exemples dans l'Ancien Testament » $(\mathrm{I} ; 14)$.

(43) Parmi elles figure sans surprise Lequinio dont il recopie, le 17 janvier 1794, l'adresse aux citoyens de Vendée, de Charente-Inférieure et des Deux-Sèvres, sous la rubrique Blasphème abominable ainsi conclue : «Homme diabolique, misérable vomi par l'enfer, ne crains pas, la terre s'entrouvre pour t'engloutir. Tu fais la guerre à Dieu et à sa religion. Es-tu donc un de ces antéchrist qui doivent tromper les hommes ».

(44) De ceux des grandes figures de la Révolution (Dufriche-Valazé, Condorcet), à ceux des figures notables havraises, comme l'ancien intendant de la marine Mistral ou le négociant suisse Joseph Roth dont il recopie intégralement les deux lettres d'adieu le 30 mai 1797, la présence du suicide jalonne les pages du Précis.

(45) Les deux ouvrages (Mon agonie de 38 heures ou récit de ce qui m'est arrivé de ce que j'ai vu et entendu pendant ma détention dans la prison de l'abbaye de Saint-Germain depuis le 22 août jusqu'au 4 septembre et Almanach des honnetes gens écrit par. S. Maréchal, "contenant des prophéties pour chaque mois de l'année 1793, des anecdotes peu connues sur les journées des 10 aout, 2, 3 septembre 1792 et la liste des personnes égorgés dans les différentes prisons) sont respectivement écrits par Jourgniac Saint- Méard qui, arrêté le 22 août et emprisonné à l'abbaye, est le témoin oculaire 
l'occasion d'une Fête célébrée au Havre ${ }^{46}$ pour stigmatiser les blasphèmes du représentant en mission Lacroix parlant dans la chaire de l'église NotreDame (où Toussaint Bonvoisin pouvait entendre ces prêtres condamnés à l'exil anglais et dont il admirait la pastorale), et enfin pour commenter les paroles d'apostasie d'un prêtre abdicataire, expriment les moments de désarroi pendant lesquels il faillit sombrer dans un renoncement coupable que seule pouvait éviter la facilité de recopier la presse.

Pour faire face à la tentation du silence, se retirer derrière un article du journal est ainsi, avant toute autre chose, la solution pour dire l'indicible. À défaut d'exposer les responsabilités des philosophes, de relater l'insurrection fédéraliste ou la tragédie de Saint-Domingue, dont les enjeux sont trop importants pour être l'objet d'une analyse, ce retrait offre la possibilité, en permettant d'écrire sans la difficulté d'exposer son propre argumentaire, par le biais des lettres d'abdication ${ }^{47}$, de la sécheresse des listes d'exécutés ${ }^{48}$ ou des bilans mensuels des condamnés par le tribunal révolutionnaire ${ }^{49}$, de réaliser, a minima, les devoirs d'instruire et de mémoire qui sont ceux de Toussaint Bonvoisin. Cette pratique, devenue systématique avec le début de la déchristianisation, ne prend pas fin avec la chute de Robespierre qu'il relate longuement (XXVIII ; 25/52). La crainte du retour de la Terreur est un sentiment qui taraude Bonvoisin pendant de longs mois et, dans ce contexte, se retirer derrière le texte reste un moyen pour parvenir à écrire, comme en témoignent les portraits de Robespierre directement inspirés des journaux et des pamphlets thermidoriens ${ }^{50}$ qu'il dresse les $1^{\text {er }}$ août et 4 août 1794, ou les longues pages de description des horreurs du Luxembourg qu'il veut « livrer à l'histoire et à l'indignation de la postérité » (XIX ; 57) en se retirant derrière le discours de Real au Club des jacobins ${ }^{51}$. Il faut attendre « le coup fatal porté aux Jacobins », auquel il consacre rien moins de 20 pages, le 17 octobre 1794 (XXI ; 87/93 ; XXII

des massacres et Sylvain Maréchal qui commence à relater les massacres à partir de la page 23 de l'ouvrage indiqué.

(46) Il s'agit de la Fête du Repos qu'il relate le 21 août 1793.

(47) Toussaint Bonvoisin recopie dix lettres d'abdication entre le 21 octobre 1793 (lettre de Le Masson, devenu assesseur du juge de paix de Beaurepaire ; VII/72) et le 10 février 1794 (lettre de Trocumatrix lors du passage d'Albitte à Bourg-en-Bresse, XII/26).

(48) Plus de 200 personnes condamnées par le tribunal populaire sont mentionnées dans le Précis entre le 7 décembre 1793 (le député Kersaint) et le 3 mars 1796 (les compagnons de Stofflet).

(49) On recense la copie de 39 bilans des exécutions prononcées par le tribunal populaire.

(50) Michel BIARD, « Après la tête, la queue ! La rhétorique antijacobine en Fructidor an II-Vendémiaire an III », dans Michel VoveLLE (dir.), Le tournant de l'an III. Réaction et terreur blanche dans la France révolutionnaire, Paris, CTHS, 1997, p. 201-213.

(51) Il s'agit d'André Réal, ancien accusateur public près du tribunal extraordinaire puis substitut du procureur de la commune de Paris par Danton. 
1/12) » pour que s'éloigne, sans jamais disparaître, une pratique du journal fondée sur la copie d'articles pour aider à résister par l'écrit.

L'effacement progressif de ce rôle permet de mieux saisir l'instrumentalisation de la revue de presse par Toussaint Bonvoisin à des fins pédagogiques qui est l'autre aspect de la mobilisation du journal. Cette instrumentalisation émerge lorsque, après quelques mois de tâtonnements, entre janvier et l'automne 1793, le mercier choisit définitivement un mode de narration didactique qui rompt avec ce récit originel commencé sans ordonnancement, dans lequel la relation des grands événements parisiens côtoie sans logique des réminiscences havraises empreintes de nostalgie et des explosions de colère fortement déconstructrices pour le récit. À l'automne 1793, en l'espace de deux mois ${ }^{52}$, il choisit une présentation systématique mensuelle dont l'efficacité repose sur la présence de deux rubriques qui interrompent une présentation continue des événements.

Or le choix de ce mode narratif consacre la reconnaissance de la valeur pédagogique du journal auquel Toussaint Bonvoisin reconnaît d'ailleurs ouvertement les vertus du pouvoir de dire la vérité et de faire des synthèses ${ }^{53}$.

Exceptées les «Réflexions », le premier type de rubriques qui, partant d'un temps fort de la crise, développent dans une quinzaine de pages et en employant la méthode de la controverse chère à Bossuet les positions morales et spirituelles du mercier, c'est en effet le contenu des articles parcourus lors de la revue de presse quotidienne qui est le point d'appui principal fondant la valeur didactique de ce récit.

L'utilisation pédagogique de la presse est reconnue par le choix de copier des articles brefs, le plus souvent extraits des «Variétés » ou des " Nouvelles étrangères », pour précéder et succéder aux "Réflexions », lesquelles sont les véritables antidotes au poison instillé par les pratiques

(52) Le 30 octobre, après l'épuration de la municipalité havraise soupçonnée de sympathie fédéraliste, Toussaint Bonvoisin met en place pour la première fois une rubrique appelée «Faits particuliers arrivés au Havre » dans laquelle il fait la synthèse de la vie politique sociale et religieuse au Havre (VIII ; 1/5). En novembre 1793, les 21 et 30 du mois, émerge une deuxième rubrique intitulée « Réflexions », dans laquelle il tire les leçons qu'il convient de la crise sur le plan spirituel (IX ; 70/81 et $\mathrm{X} ; 42 / 51)$.

(53) La croyance de Bonvoisin dans le pouvoir de vérité émerge avec force dans son commentaire du coup d'état du 18 Brumaire auquel il consacre 30 pages : " l'abondance des matières ne nous permet de donner les faits dans toutes leurs étendues. C'est pourquoi nous joindrons les journaux les plus exacts et les plus véridiques à cette partie » (XXXXII ; 22/23). Sa croyance dans le pouvoir de synthèse peut être illustrée avec ce commentaire de la copie du discours pacifique de Lord Lansdawne le 10 mars 1794 : « dans cette douzième partie se trouve le discours de Lord Lansdawne tendant à la paix. Cette pièce donne une idée du résultat de ce qui s'est passé dans les premières campagnes de guerre avec les puissances coalisées contre la France ». 
révolutionnaires. L'information journalistique constitue donc le cœur du récit, donnant la matière nécessaire à la mise en place des « Réflexions ». Mais sa place est surtout valorisée par la construction d'une seconde rubrique : les «Faits particuliers arrivés au Havre ». À la différence de la première, les « Réflexions » qui jalonnent le texte au gré des événements, celle-ci est introduite chaque dernier jour du mois. Toussaint Bonvoisin y propose, en compilant des informations sur la Révolution au Havre qu'il sélectionne dans le Journal de Rouen et dans Le Courrier Maritime ${ }^{54}$, une lecture originale de la crise telle qu'elle se serait déroulée dans cette ville. Cette lecture, en raison des choix opérés par Toussaint Bonvoisin, tend à valoriser les résistances religieuses à la Révolution et le fort sentiment patriotique des habitants du Havre dont il souhaite renforcer leur conscience de devoir défendre les intérêts nationaux face à la menace anglaise en raison de l'expérience historique qui est celle de cette ville depuis sa fondation ${ }^{55}$.

Les « Faits particuliers au Havre » montrent une sélection de l'information qui est l'autre aspect de l'instrumentalisation pédagogique du journal par Toussaint Bonvoisin. Pour faire prendre conscience de ce devoir patriotique, le mercier fait d'ailleurs, au-delà de la seule analyse des « Faits particuliers », une sélection des articles qui est fondée sur l'idée de surreprésenter les Nouvelles de l'Angleterre ${ }^{56}$. C'est en effet le tiers des pages réservées aux « Nouvelles Etrangères » qu'il consacre, dès l'an II, à ces informations qui n'englobent que $6 \%$ des informations étrangères dans le Journal de Rouen ${ }^{57}$. On remarque par la suite leur montée irrésistible jusqu'à la fin de l'écriture du Précis ${ }^{58}$.

Cette sélection des informations sert d'autres intentions. Parmi elles, prolongeant la perspective précédente, émerge la volonté de construire un récit destiné à placer l'évolution du Havre sous la Révolution dans le cadre

(54) Le Courrier Maritime est la seule feuille havraise. Bien que diffusée au Havre avant la Révolution, ce quotidien qui consacre ses colonnes à la description des mouvements touchant le port du Havre est très peu utilisé par Toussaint Bonvoisin. Peu intéressé par les questions économiques, il préfère recourir aux informations diffusées dans le Journal de Rouen sur la vie politique et religieuse au Havre.

(55) Sur cet aspect identitaire, voir : Jean-Baptiste GASTINNE, « Le Havre du XVI ${ }^{\mathrm{e}}$ au XVIII siècle. Genèse d'une ville et d'une population urbaine », Thèse de doctorat de l'université de Paris IV préparée sous la direction de Jean-Pierre BARDET, 2008, 3 volumes

(56) On soulignera d'ailleurs que le discours pacifique prononcé par le député libéral Lord Lansdawne devant le Parlement anglais est le discours le plus long reproduit dans le Précis. Il occupe près de trente pages du douzième cahier (p. 45-73).

(57) Plus des deux tiers du $38^{\mathrm{e}}$ cahier sont consacrés aux opérations navales avec l'Angleterre, au point de conduire Toussaint à arrêter ses relations mensuelles des Faits particuliers arrivés au Havre.

(58) Elles représentent en effet plus de la moitié des pages des Nouvelles étrangères après le coup d'État du 18 Fructidor an V, remplissant même la quasi-totalité du $38^{\mathrm{e}}$ cahier écrit entre mai et septembre 1798. 
d'une histoire commune aux villes portuaires maritimes. Dans le journal de Toussaint Bonvoisin, on remarque en effet, face à l'indifférence qu'il manifeste devant la description d'événements qui se sont déroulés dans les villes voisines - il est très peu de pages sur la révolution à Rouen ou sur l'insurrection caennaise du printemps 1793-, les longs développements consacrés à la mobilisation de la marine à Brest, à la défense de Toulon, aux troubles politiques de Marseille et aux violences de la Terreur à Nantes. Si l'on ajoute les 700 pages que Toussaint Bonvoisin consacre aux « Faits Particuliers au Havre », l'histoire de la révolution dans les ports maritimes pendant la Révolution, une histoire très attentive aux désastres provoqués par le ralentissement de l'activité du négoce et la défense des intérêts coloniaux, occupe de loin (31\%) la part la plus importante des informations réservées à la province.

La sélection lui permet aussi, pour donner une vision moins noire de l'humanité que celle reflétée par les « Réflexions » dont la fonction première est de stigmatiser la responsabilité des Philosophes dans le déclenchement de la Révolution, de valoriser le poids des résistances à la Révolution. La place occupée par l'insurrection de Vendée, dont il désapprouve le soulèvement, recouvre ainsi, avec un tiers des pages en l'an II, une place qui dépasse celle qui lui est réservée dans le Journal de Rouen $^{59}$. La sélection de l'information lui permet enfin de se rapprocher de la construction d'une histoire quasi-positiviste, fondée sur la connaissance des textes législatifs de la Révolution. On voit ainsi Toussaint Bonvoisin recopier les textes entiers de 80 décrets révolutionnaires.

La familiarisation avec la lecture quotidienne du journal, que la revue de presse serve à résister à la tentation du silence ou à des fins pédagogiques, n'est pas sans conséquences. L'un des intérêts du Précis réside dans la possibilité d'observer les modifications qu'elle peut entraîner s'agissant du rapport liant l'individu avec la politique durant la crise révolutionnaire (annexe 3). Il y a ainsi une différence de taille dans la façon dont Toussaint Bonvoisin appréciait et travaillait à l'été 1793 sur l'article de Robespierre publié dans le Défenseur de la Constitution ${ }^{60}$, et le discrédit et l'ignorance dans lesquels il tient les journaux gouvernementaux qui prétendent défendre la politique du Directoire à partir de l'automne 1795, et plus encore après la crise fructidorienne. Alors qu'en août 1793, Toussaint Bonvoisin utilisait abondamment la feuille robespierriste, lui reconnaissant même, tout en

(59) En l'an II $20 \%$ du Journal de Rouen est occupé par la Vendée et la Chouannerie. Voir Éric WAUTERS, Une presse... op. cit. p. 381-386.

(60) Voir la note 16. 
détestant son auteur et le manque d'application des idées défendues (dont la liberté de la presse), une justesse de principes qui lui permettait d'en faire le socle de sa réflexion pour justifier l'acceptation de la Constitution de 1793, deux ans plus tard, les journaux républicains, qu'ils soient parisiens ou rouennais, sont absents de l'écriture de son journal dans lequel il témoigne ouvertement, après la crise fructidorienne, du peu d'estime dans lequel il tient les feuilles qui défendent le second Directoire. «On doute de ses nouvelles » (XXXVI; 67), «nos gouvernans font-ils courir ce bruit pour voir comment il sera pris par le peuple », « on a quelques raisons de croire à l'exactitude des détails » (XXXVII ; 4), les remarques qu'il distille dans les marges de son texte en décembre 1797 sont nombreuses à témoigner de cette défiance qui montre, derrière l'envie d'instruire, la naissance d'une forme de militantisme.

À cette défiance nouvelle répond une confiance dans la presse conservatrice qui le conduit, lors des débats sur le vote de la Constitution de l'an III et de l'Acte additionnel des Cinq Cents, à modifier la structure de la rubrique «Réflexions » qui était depuis l'automne 1793 le point d'appui principal pour l'expression de ses idées personnelles. Elle devient en effet, en trois mois, l'objet d'une modification marquée par un retrait derrière le texte du journaliste qui est le signe fort de l'influence de la presse royaliste. La rupture est amorcée le 20 juin 1795 lorsque l'on voit Toussaint Bonvoisin se retirer derrière un écrit qu'il attribue au journaliste Bresson $^{61}$ pour prévenir des dangers que pourrait comporter l'adoption d'une constitution trop démocratique (XXVII ; 90/94). Les 25 et 29 août 1795, ayant perdu ses derniers espoirs de voir voter une Constitution défendant des principes raisonnables avec le vote du décret des Deux Tiers

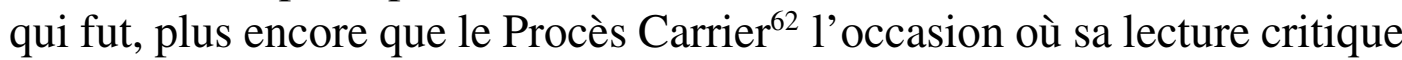
de la presse conservatrice avait déjà suscité de vives réserves à l'égard de la politique de la Convention, ce sont tout simplement les éditoriaux du Messager du Soir (XXVIII ; 76/84 et XXIX : 6-9) qui se chargent de développer les idées personnelles de Toussaint Bonvoisin.

Il est difficile de tirer des conclusions définitives à partir de cette étude de cas qui, à notre connaissance, n'a pas d'équivalent. Toutefois, elle

(61) Il s'agit de Jean-Baptiste Bresson (1760-1832), ancien député des Vosges, dont Toussaint Bonvoisin recopie le brûlot écrit contre la Constitution de 1793 au moment où sont discutées les bases de la Constitution de l'an III. Le texte, Réflexions sur les bases d'une constitution, est en fait écrit par Viénot de Vaublanc.

(62) Sur cet aspect, Éric SAUNIER, «Le négoce et la Terreur : les apports du Précis de François-Toussaint Bonvoisin », dans Michel BIARD (dir.), Les politiques de la Terreur, 1793-1794, Rennes, Presses universitaires de Rennes, Collection Histoire, 2008, p. 367-379. 
permet de faire deux réflexions s'agissant des potentialités que peut offrir la réouverture du chantier des égo-documents de la période révolutionnaire. La première, revenant à notre constat initial sur les carences de l'étude de la presse durant les années 1789-1799, est la capacité des écrits du for privé de nous faire saisir des formes et des objectifs nouveaux d'appropriation du journal. Face au regard presque exclusif que les chercheurs portent sur ce phénomène dans les lieux d'expression collective (clubs, ateliers...), l'un des mérites des études sur les pratiques journalistiques dans le secret des cabinets privés est de montrer une utilisation du journal différente, à des fins non politiques, dont l'importance mériterait d'être mesurée. Avec ces fins non politiques qu'est l'usage d'une revue de presse pour faciliter la résistance par l'écriture et l'instrumentalisation à des fins éducatives, ce type d'approche, en témoignant, comme c'est le cas de ce journal à partir du Directoire, d'une «mobilisation politique individuelle», offre aussi des éclairages non négligeables sur la formation de la culture politique de la génération suivante qui, dans le camp conservateur auquel appartient Bonvoisin, choisit d'en découdre ouvertement avec la France Libérale, comme ce fut le cas ses deux fils les plus âgés Louis et Benjamin ${ }^{63}$.

À l'évidence, quand, retenant l'enseignement paternel, Louis, véritable «thuriféraire de la Restauration ${ }^{64}$, et Benjamin ${ }^{65}$, peintre de la régénération spirituelle, prennent leur plume et leur pinceau pour s'engager publiquement, à la différence de leur père qui, malgré la mobilisation de la période du Directoire, resta toute sa vie en dehors de l'arène politique ${ }^{66}$, la revue de presse pratiquée par ce dernier avait joué un rôle de première importance dans le choix de cet engagement.

(63) Éric Saunier, « Les papiers... », art. cit., p. 163-177.

(64) Voir Corinne Legoy, L'enthousiasme désenchanté. Eloge du pouvoir sous la Restauration, Paris, SER, 2010. Louis-Toussaint Bonvoisin (1785-1824), le fils aîné du mercier Toussaint Bonvoisin, est l'auteur de poèmes élogieux envers les Bourbons et d'écrits moralistes dont nous avons reproduit quelques exemples (Éric Saunier, « Les papiers », art. cit., p. 173-177).

(65) Peintre de la régénération spirituelle sous la Restauration, Benjamin Bonvoisin (17881860), second fils de Toussaint Bonvoisin, est le donataire des papiers écrits par son père au premier bibliothécaire de la ville de Montivilliers, Charles Blanchet, en 1859.

(66) Toussaint Bonvoisin a en effet pour seule implication dans la cité de faire son service dans le garde nationale du Havre. 


\section{Annexe 1}

La présence du journal dans les écrits de Toussaint Bonvoisin

\begin{tabular}{|c|c|c|c|c|}
\hline \begin{tabular}{|l} 
Partie \\
Précis
\end{tabular} & du Dates de rédaction & Journaux & $\begin{array}{l}\text { Pages } \\
\text { isolées }\end{array}$ & Articles \\
\hline 18 & 18/07-6/08/1794 & 2 & & \\
\hline 19 & $7-30 / 08 / 1794$ & & & \\
\hline 20 & $31 / 08-24 / 09 / 1794$ & 1 & & \\
\hline 21 & 25/09-18/10/1794 & & & \\
\hline 22 & $18 / 10-10 / 10 / 1794$ & & & \\
\hline 23 & $11 / 11-5 / 12 / 1794$ & & & \\
\hline 24 & $\begin{array}{l}6 / 12 / 1794- \\
10 / 01 / 1795\end{array}$ & & & 2 \\
\hline 25 & $10 / 01-15 / 02 / 1795$ & & 3 & 1 \\
\hline 26 & $16 / 02-29 / 04 / 1795$ & 3 & 5 & 3 \\
\hline 27 & $1 / 05-21 / 06 / 1795$ & 3 & & 1 \\
\hline 28 & $22 / 06-25 / 08 / 1795$ & 6 & 2 & 2 \\
\hline 29 & 27/08-20/09/1795 & 5 & & \\
\hline 30 & 21/09-17/11/1795 & 6 & 3 & 3 \\
\hline 31 & $\begin{array}{l}18 / 11 / 1795- \\
30 / 01 / 1796\end{array}$ & 12 & 7 & 2 \\
\hline 32 & 1/02/-30/04/1796 & 11 & 2 & 5 \\
\hline 33 & $1 / 05-28 / 07 / 1796$ & 8 & 7 & 4 \\
\hline 34 & $\begin{array}{l}1 / 08 / 1796- \\
28 / 04 / 1797\end{array}$ & 27 & 8 & 1 \\
\hline 35 & $1 / 05-5 / 05 / 1797$ & 12 & 8 & 2 \\
\hline 36 & 6/08/-30/11/1797 & 24 & 8 & 1 \\
\hline 37 & $\begin{array}{l}1 / 12 / 1797- \\
12 / 04 / 1798\end{array}$ & 11 & 2 & 1 \\
\hline 38 & $\begin{array}{l}15 / 04 / 1798- \\
5 / 09 / 1798\end{array}$ & 4 & 8 & 1 \\
\hline 39 & $\begin{array}{l}\text { 10/09/1798- } \\
23 / 01 / 1799\end{array}$ & 13 & 5 & 4 \\
\hline 40 & 25/01/-13/04/1799 & 11 & 5 & \\
\hline 41 & $14 / 04-23 / 10 / 1799$ & 16 & 5 & 3 \\
\hline
\end{tabular}




\begin{tabular}{|lllll|}
42 & $24 / 10 / 1799-$ & 45 & 10 & 3 \\
43 & $10 / 11 / 1800$ & & & \\
& $16 / 11 / 1800-$ & 5 & 6 & \\
44 & $4 / 12 / 1801$ & & & \\
& $23 / 12 / 1801-$ & 34 & 1 & \\
& $12 / 01 / 1803$ & & & \\
\hline
\end{tabular}


Annexe 2

Les sources journalistiques de l'écriture du Précis (novembre 1792-janvier 1803)

\begin{tabular}{|c|}
\hline $\begin{array}{l}\text { Sources principales de l'écriture } \\
\text { Novembre 1792- Octobre } 1793\end{array}$ \\
\hline Chronique nationale et Etrangère (07/1790-01/1793) \\
\hline Dir : Jacques Leclerc \\
\hline Sensibilité politique: journal conservateur, \\
\hline tendance feuillantine \\
\hline Journal de Rouen (1791-1799) \\
\hline Dir : J. -B. Milcent, Noël de la Morinière (02/1793) \\
\hline $\begin{array}{l}\text { Sensibilité politique : journal patriote,tendance modérée (rejet de la } \\
\text { République }\end{array}$ \\
\hline Journaux parisiens utilisés entre octobre et novembre 1792 : \\
\hline Journaux royalistes : Le Postillon de la Guerre, Le Messager du Soir \\
\hline $\begin{array}{c}\text { Journaux patriotes : Le Patriote français, Le Défenseur de la } \\
\text { Constitution }\end{array}$ \\
\hline Novembre 1793-Janvier 1795 \\
\hline Annales de la Révolution, (11/1793-05/1795 \\
\hline Dirs : Adr. Dubosc \& Guilbert, \\
\hline exilé en Suisse \\
\hline Tendance : Journal passé à la Gironde, modéré \\
\hline Journal de Rouen (1791-1799) \\
\hline Dir : Noël de la Morinière \\
\hline Journal attentiste en l'An II \\
\hline Octobre 1794- Septembre 1797 \\
\hline L'Observateur de l'Europe (01/-10/1795) \\
\hline L'Eclipse (10/1795-03/1796) \\
\hline
\end{tabular}




\begin{tabular}{|c|}
\hline L'Observateur $(03 / 1796-10 / 1797)$ \\
\hline Dir : Magloire Robert \\
\hline Tendance : royaliste \\
\hline Directoire \\
\hline Journal de Rouen (1791-1799) \\
\hline Dir : Noël de la Morinière \\
\hline Tendance :Journal républicain modéré \\
\hline Septembre 1797-novembre 1799 \\
\hline La Gazette Historique (09/1797-11/1799) \\
\hline Le Bien informé (09/1797-11/1799), \\
\hline Tendance : royaliste \\
\hline Journal de Rouen (1791-1799) \\
\hline Dir : Noël de la Morinière \\
\hline Tendance : Journal républicain modéré \\
\hline Novembre 1799- janvier 1803 \\
\hline Affiches \& annonces de Rouen \\
\hline ou Journal d'indication de Politique, Littérature \\
\hline Sciences et Arts et du commerce \\
\hline$(04 / 1798-06 / 1799)$ \\
\hline (Dir : N. Martin) \\
\hline Journal Politique, Littéraire, du Commerce, \\
\hline Sciences et Arts \\
\hline (Affiches \& annonces de Rouen) \\
\hline$(06 / 1799-11 / 1799)$ \\
\hline (Dir : Robert -sous le nom de Thomas) \\
\hline Les Chroniques de l'Europe \\
\hline$(11 / 1799-08 / 1810)$ \\
\hline (Dir : Robert -sous le nom de Thomas) \\
\hline Sensibilité politique : journaux royalistes \\
\hline
\end{tabular}


Annexe 3

Toussaint Bonvoisin et l'utilisation de la presse de la réaction thermidorienne au Consulat

\begin{tabular}{|c|c|c|c|c|c|c|c|c|c|}
\hline $\mathbf{N}^{\circ}$ cahier & Dates de rédaction & $\mathbf{I}$ & II & III & IV & $\mathbf{V}$ & VI & VII & VIII \\
\hline 18 & 18/07-6/08/1794 & & & & & & & & \\
\hline 19 & 7-30/08/1794 & 1 & & & & & & & \\
\hline 20 & $31 / 08-24 / 09 / 1794$ & & 1 & & & & & & \\
\hline 21 & 25/09-18/10/1794 & & & & & & & & \\
\hline 22 & 18/10-10/10/1794 & & & & & & & & \\
\hline 23 & 11/11-5/12/1794 & & & & & & & & \\
\hline 24 & 6/12/1794-10/01/1795 & & & & & & & & \\
\hline 25 & 10/01-15/02/1795 & & 4 & & & & & & \\
\hline 26 & $16 / 02-29 / 04 / 1795$ & & 3 & 5 & & & & & \\
\hline 27 & 1/05-21/06/1795 & & 3 & & & & & & \\
\hline 28 & 22/06-25/08/1795 & & 1 & 6 & & & & & \\
\hline 29 & 27/08-20/09/1795 & & 1 & 3 & & & & & \\
\hline 30 & 21/09-17/11/1795 & & 4 & 2 & & & & & \\
\hline 31 & 18/11/1795-30/01/1796 & & 10 & 1 & & & & & \\
\hline 32 & 1/02/-30/04/1796 & & & 16 & & & & & \\
\hline 33 & 1/05-28/07/1796 & & & 13 & & & & & \\
\hline 34 & 1/08/1796-28/04/1797 & & & 17 & & & & & \\
\hline 35 & 1/05-5/05/1797 & & & 5 & 21 & & & & \\
\hline 36 & 6/08/-30/11/1797 & & & & & & & & \\
\hline 37 & 1/12/1797-12/04/1798 & & & & 17 & & & & \\
\hline 38 & 15/04/1798-5/09/1798 & & & & 8 & 1 & & & \\
\hline 39 & 10/09/1798-23/01/1799 & & & & 4 & 4 & 5 & 7 & \\
\hline 40 & 25/01/-13/04/1799 & & & & & 11 & 12 & 6 & \\
\hline 41 & 14/04-23/10/1799 & & & & & 2 & & & 17 \\
\hline 42 & 24/10/1799-10/11/1800 & & & & & & & & 14 \\
\hline 43 & $16 / 11 / 1800-4 / 12 / 1801$ & & & & & & & & 16 \\
\hline 44 & 23/12/1801-12/01/1803 & & & & & & & & \\
\hline
\end{tabular}

I : Annales de la Révolution

II : Journal de Rouen 
III : L'Observateur de l'Europe, L'Observateur à Rouen

IV : L'Eclipse

$V:$ Gazette Historique et littéraire

$V I$ : Le Bien Informé

VII : L'Ami des Lois

VIII : Chronique de l'Europe

Les chiffres indiqués englobent exclusivement la présence des numéros entiers ou de moitiés de ces numéros.

Éric SAUNIER Normandie université / université du Havre UMR Idees 6266 (CIRTAI), 25 rue Philippe Lebon 76063 Le Havre cedex eric.saunier@wanadoo.fr 